\title{
Gyrokinetic analysis of radial dependence and global effects on the zero particle flux condition in a TCV plasma
}

\author{
A. Mariani $,{ }^{1}, *$ S. Brunner, ${ }^{2}$ G. Merlo, ${ }^{3}$ and O. Sauter ${ }^{2}$ \\ ${ }^{1}$ Istituto di Fisica del Plasma IFP-CNR, Via R. Cozzi 53, 20125 Milano, Italy \\ ${ }^{2}$ École Polytechnique Fédérale de Lausanne (EPFL), Swiss Plasma Center (SPC), 1015 Lausanne, Switzerland \\ ${ }^{3}$ University of Texas at Austin, Austin, TX 78712, USA
}

(Dated: January 28, 2019)

\begin{abstract}
In small-sized tokamaks, finite Larmor radius effects could lead to a significant discrepancy between gyrokinetc local flux-tube results and global ones. This has been highlighted by previous turbulent transport studies as in [B. F. McMillan et al., Phys. Rev. Lett. 2010]. The impact of such effects on the zero particle flux condition is investigated here. The zero particle flux condition is useful to estimate the density peaking, reducing the uncertainty on physical input parameters derived from experimental measurements, for cases where the particle source is negligible. This constraint has been applied to the analysis of a particular TCV discharge, where a detailed reconstruction of the zero particle flux hyper-surface in the multidimensional physical parameter space at fixed radius had been presented in [Mariani et al., Phys. Plasmas 2018]. Here, we extend these results, investigating their radial dependence, together with the impact of global effects. These so called $\rho^{*}$ effects are analysed by simulating a plasma annulus corresponding to the stiff region $0.4<\rho_{\text {tor }}<0.8$. Because of the computational cost of the non-linear global gyrokinetic simulations, we restrict to a two species plasma in the collisionless regime, with heavy electrons and simplified density and temperature radial profiles. With these simplifications, the results seem to point towards global effects on the zero particle flux condition being relatively weak.
\end{abstract}

\section{INTRODUCTION}

The understanding of heat, particle and momentum transport remains one of the main topics in contemporary magnetic plasma confinement community. The production of peaked density and temperature profiles in tokamaks is needed to achieve the required fusion performances in the next generation experiments such as ITER [1] and DEMO [2]. Gyrokinetic $(\mathrm{GK})$ simulations are a powerful tool to study the mechanisms that underlie turbulent transport. There are two main kinds of GK simulations: flux-driven (FD) simulations and gradient-driven (GD) ones. The former are run specifying the input fluxes, letting the density and temperature profiles relax in time, while in the latter the density and temperature profiles are set as input and the fluxes are computed as output. FD simulations are more realistic since the sources are imposed in the experiments, i.e. the fluxes. GD simulations, on the contrary, are hardly comparable with experiments when stiff profiles are present, i.e. when a small variation of the gradients within the experimental error bars leads to a large variation of the fluxes. Unfortunately, FD simulations are computationally demanding, since they need to simulate over transport time scale, in order to let the profiles relax, and it is rarely possible to afford them with the available resources.

In order to make GD simulations reliable, overcoming the problem of profile stiffness, the values of the main turbulence drivers, such as the density and temperature gradients of the different plasma species, the electron to ion temperature ratio, the main impurity concentration and gradient, all have to be known with very good accuracy. Unfortunately, these experimentally derived inputs are usually only provided

\footnotetext{
* Electronic address: mariani@ifp.cnr.it
}

with relatively large error bars. These uncertainties can be reduced by enforcing physical constraints. The zero particle flux constraint, consisting in enforcing zero average particle flux at each magnetic surface for every plasma species, can be considered for discharges with no external particle injection. Identifying the hyper-surface satisfying this condition in the multi-dimensional parameter space, and in particular the evaluation of the peaking factor (PF), that is the electron density gradient corresponding to the simultaneous vanishing of the particle fluxes for all species, is in itself a topic of research [3-7].

A detailed characterisation of the zero particle flux hypersurface has been performed by the authors of this work in [8], considering a discharge of the Tokamak à Configuration Variable (TCV) [9] at the Swiss Plasma Center (SPC-EPFL, Lausanne, $\mathrm{CH}$ ), published in [10, 11], of interest for momentum transport analysis. This discharge features a toroidal rotation reversal during a density ramp up, occurring in conjunction with a relatively small change in the plasma density. The main body of the analysis presented in [8] has been performed selecting a snapshot close to the reversal, and it is restricted to a single radial position $\rho_{\text {tor }}=0.6$, i.e. close to midradius, where $\rho_{\text {tor }}$ is the normalised radial position defined as $\rho_{\text {tor }}=\sqrt{\Phi / \Phi_{\text {edge }}}$, where $\Phi$ is the toroidal magnetic flux. Linear and nonlinear (NL) flux-tube GK simulations have been carried out, finding sets of zero particle flux-matching parameters (including $\mathrm{PF}$ ) in both collisionless and collisional regime, also evaluating the effect of the carbon impurity. Note that flux-tube simulations are inherently GD.

The first part of the present work consists in the investigation of the radial dependence of the PF which has been evaluated in [8], restricting for simplicity to a two species plasma in the collisionless regime. Density gradient scans of linear GK simulations have been performed with the local (fluxtube) version of the GENE code [12], allowing the evalua- 
tion of the $\mathrm{PF}$ at five selected radii in the stiff radial region $\rho_{\text {tor }} \sim[0.4,0.8]$ [13]. Considering constraints on flux ratios allows us to get quick predictions of the fluxes by quasilinear (QL) estimates, where only ratios are practically available from linear simulations. NL simulations have been performed for a subset of cases to test the QL estimates.

The second part of the paper is about the evaluation of the global effects on the PF. Indeed, finite machine size effects requiring so-called global simulations are known to be important in smaller size tokamaks like TCV [14-18]. Global effects are related to the finite value of $\rho^{*}=\rho_{i} / a$, where $\rho_{i}$ is the ion Larmor radius and $a$ is the minor radius of the tokamak. This value is relatively important in case of smaller-sized tokamaks such as TCV, where typically $\rho^{*} \sim 1 / 80$. The smaller $1 / \rho^{*}$ the bigger the overestimation of the fluxes from flux-tube compared to global simulations. Due to their high computational cost, these global simulations have been carried out restricting the radial domain to the stiff $\rho_{\text {tor }} \in[0.4,0.8]$ region and considering reduced physics. The heavy electrons approximation, which consists in considering a smaller deuteron-electron mass ratio $\left(m_{i} / m_{e}=400\right.$ instead of physical $m_{i} / m_{e}=3672$ ), has been adopted. Nevertheless, the impact of this approximation has been found to be negligible. Moreover, analytical density and temperature profiles with constant logarithmic gradients have been used, which are consistent with the local experimental values at $\rho_{\text {tor }}=0.6$.

The paper is organised as follows: in section II the experimental setting, together with the simulations parameters, are introduced. A description of the QL model which has been used to evaluate the fluxes starting from the linear simulations is presented in section III. Section IV A contains the QL analysis of the radial variation of the PF. The PF is found to appreciably vary for $\rho_{\text {tor }}<0.6$, remaining almost constant for larger radii. In the following section IV B, the QL results are compared with NL ones in a subset of cases. The very good QL-NL agreement validates the previous QL analysis. Section $\mathrm{V}$ contains the study of the global effects on the PF. It is found that, even if the particle flux is affected by global effects, the $\mathrm{PF}$ is not. Finally, conclusions are drawn in section VI. In the two appendices A and B, the dependence of the global results on the strength of the Krook-type heat and particle sources and the global effects on the particle flux to ion heat flux ratio are presented respectively. Quite remarkably, the ratio of the particle flux to the ion heat flux is found not to vary, when both going from local to global simulations and when increasing the strength of the Krook-type heat and particle sources. We plan in the future to generalise the results of this paper, exploring the collisional regime, investigating the carbon impurity effect and looking at the impact of considering the true experimental profiles as input for the global simulations.

\section{EXPERIMENTAL SETUP AND SIMULATION PARAMETERS}

In this work we analyse TCV shot \#28355, which is a limited ohmic L-mode, presenting a electron-deuteron plasma, with carbon as main impurity (same discharge as considered in $[8,10,11])$. The electron density $n_{e}$ and temperature $T_{e}$ are measured experimentally with Thomson scattering diagnostic, while carbon density $n_{c}$ and temperature $T_{c}$ are given by charge exchange recombination spectroscopy (CXRS). The corresponding quantities for ions are obtained by enforcing neutrality $\left(n_{i}=n_{e}-6 n_{c}\right)$ and deuteron-carbon thermal equilibrium $\left(T_{i}=T_{c}\right)$. Finally, the magnetic equilibrium is recomputed using the ideal MHD solver CHEASE [19], whose output is directly interfaced to GENE. A single time slice at time $t=0.96 \mathrm{~s}$ of the discharge, just before the rotation reversal, is investigated in this work. In the analysis of the finite Larmor radius effects on the PF, the entire stiff region $0.4 \lesssim \rho_{\text {vol }} \lesssim 0.8$ [13] has been simulated using the global version of the GENE code, while in the QL analysis of the PF radial dependence only five radial positions $\rho_{\text {tor }}=0.4,0.5,0.6,0.7,0.8$ have been considered with the local flux-tube versione of GENE. Here, $\rho_{\text {vol }}=\sqrt{V / V_{\text {edge }}} \sim \rho_{\text {tor }}$, where $\mathrm{V}$ is the volume enclosed by the considered magnetic surface. The magnetic equilibrium and main plasma parameters at $t=0.96 \mathrm{~s}$ are shown in Fig.1. Figure 1 (a) shows the poloidal cross section of the magnetic equilibrium. Magnetic surfaces corresponding to the five considered radii, together with the Last Close Flux Surface (LCFS), are represented by different colors. The equilibrium magnetic field is almost up-down symmetric, as can be seen by comparing the actual magnetic surfaces with the curves obtained by flipping them with respect to the mid-plane. Elongation $\kappa$ and triangularity $\delta$ range from $\kappa=1.27, \delta=0.04$ at $\rho_{\text {tor }}=0.4$ to $\kappa=1.49, \delta=0.35$ at the LCFS. The safety factor radial profile is shown in Fig.1 (b), while density and temperature profiles are shown in Fig.1 (c) and (d), respectively. The experimental error bars are of the order of $\pm 20 \%$.

The main experimental parameters of interest for the gyrokinetic analysis, at the five analysed radial positions, are summarised in Table I. The normalised radial logarithmic gradients of the $f$ profiles $(f=n, T)$ are here defined as $R / L_{f}=$ $-\left(R_{0} / \alpha\right) d \log f / d \rho_{\text {tor }}$, where $\alpha=\sqrt{\Phi_{\text {edge }} / \pi B_{0}} \simeq 31 \mathrm{~cm}$ and $B_{0}=1.44 \mathrm{~T}$ is the vacuum magnetic field at the major radius $R_{0}=88 \mathrm{~cm}$ of the tokamak. It is worth stressing that, since for simplicity in the simulations the carbon impurity is neglected (for a detailed analysis of the impurity effect at $\rho_{\text {tor }}=0.6$ we refer to [8]), one has $R / L_{n e}=R / L_{n i}=R / L_{n}$. The experimental error bars are of order $\pm 20 \%$ on the profiles and roughly $\pm 40 \%$ on the corresponding logarithmic gradients. The other parameters are the safety factor $q$, the magnetic shear $\hat{s}=d \log q / d \log \rho_{\text {tor }}$ and the ratio of the plasma pressure to the magnetic pressure $\beta=2 \mu_{0} n_{e} T_{e} / B_{0}$, with $\mu_{0}$ the vacuum permeability.

The GENE code adopts a field-aligned coordinate system $(x, y, z)$ in the configuration space, while $\left(\mathrm{v}_{\|}, \mu\right)$ are used as velocity variables in the reduced 2-dimensional GK velocity space. Here $(x, y, z)$ represent the radial, the binormal and the parallel positions respectively ( $x=$ const $\& y=$ const define a magnetic field line, while $z$ sets the position along that line), $\mu=m v_{\perp}^{2} / 2 B$ is the magnetic moment and $\mathrm{v}_{\|}$is the parallel velocity. In the flux-tube version of the code, Fourier representation is used for both the $x$ and $y$ directions, while in the global version the Fourier representation is restricted to $y$. A typical grid size for a linear flux-tube simulation of fixed mode number $k_{y}$ with respect to the $y$ direc- 

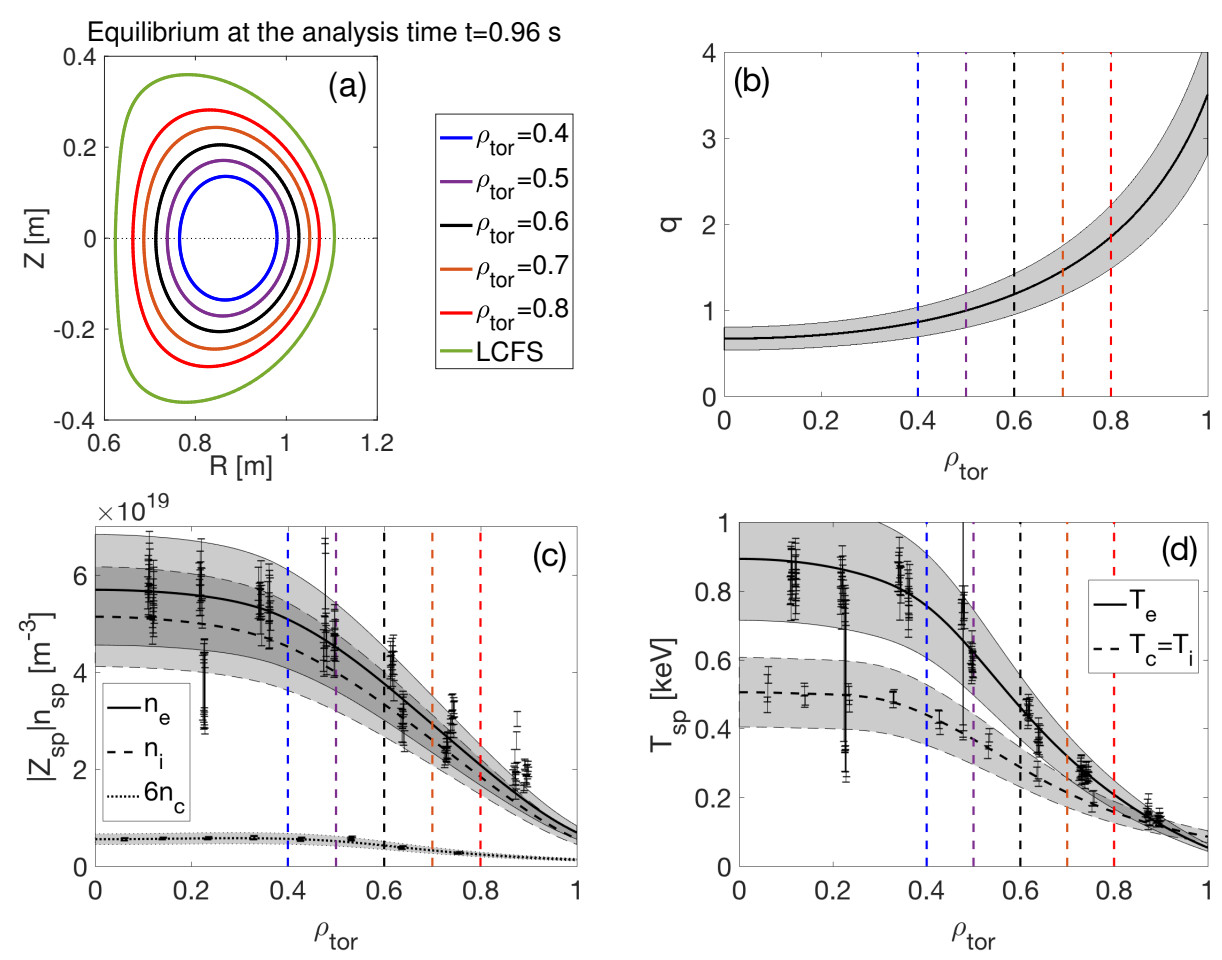

Figure 1. (color online) Magnetic equilibrium and main plasma parameters at the analysis time $t=0.96 \mathrm{~s}$. (a) CHEASE reconstruction of the poloidal cross section of the magnetic equilibrium. Magnetic surfaces corresponding to the five radii considered for the local flux-tube analysis are shown in different colors, compared with the surfaces obtained flipping them with respect to the mid-plane. (b) Safety factor profile. (c) Density profiles. $n_{\mathrm{sp}}$ are multiplied by their respective ionisation degrees $\left|Z_{s p}\right|$, satisfying neutrality $n_{e}=n_{i}+6 n_{c}$. (d) Temperature profiles. In subfigures (b)-(d) the radii of analysis are indicated by vertical dashed lines, following the same color code of (a). The $\pm 20 \%$ error bars are indicated by grey regions.

tion $\left(k_{y}=n q / \alpha \rho_{\text {tor }}\right.$, where $n$ is the toroidal mode number $)$ is $n_{k x} \times n_{z} \times n_{\mathrm{v} \|} \times n_{\mu}=48 \times 32 \times 64 \times 16$, while a typical NL simulation grid size is $n_{k x} \times n_{k y} \times n_{z} \times n_{\mathrm{v} \|} \times n_{\mu}=$ $256 \times 64 \times 32 \times 64 \times 16$. To collect sufficient statistics, the flux-tube NL simulations have been run in time up to at least $t_{\max } c_{s} / R \sim 100$, while higher values up to $t_{\max } c_{s} / R \sim 200$ when necessary. A typical grid size for a nonlinear global simulation is $n_{x} \times n_{k y} \times n_{z} \times n_{\mathrm{v} \|} \times n_{\mu}=200 \times 32 \times 32 \times 72 \times 36$. We refer to section V A for more information about the global simulation settings. Convergence tests have been performed to check the reliability of the results. In all the simulations, electrons have been treated as a self-consistent gyrokinetic species.

The flux-tube simulations have all been run retaining the real deuteron-electron mass ratio $m_{i} / m_{e}=3672$ while, in order to be feasible within the available computational resources, the global simulations have been run in the "heavy electron' approximation $m_{i} / m_{e}=400$. Nevertheless, a test has been done for a representative case (see section V), showing that the particle flux obtained using the real mass ratio is well approximated by the one given by considering heavy electrons.

\begin{tabular}{|l|c|c|c|c|c|c|c|}
\hline & $R / L_{n}$ & $R / L_{T e}$ & $R / L_{T i}$ & $T_{i} / T_{e}$ & $q$ & $\hat{s}$ & $\beta$ \\
\hline$\rho_{\text {tor }}=0.4$ & 2.65 & 4.00 & 4.02 & 0.58 & 0.86 & 0.51 & $0.74 \times 10^{-2}$ \\
\hline$\rho_{\text {tor }}=0.5$ & 4.26 & 7.21 & 6.06 & 0.60 & 0.99 & 0.8 & $0.54 \times 10^{-2}$ \\
\hline$\rho_{\text {tor }}=0.6$ & 6.15 & 9.6 & 8 & 0.62 & 1.19 & 1.15 & $0.34 \times 10^{-2}$ \\
\hline$\rho_{\text {tor }}=0.7$ & 8.28 & 11.27 & 8.69 & 0.67 & 1.46 & 1.56 & $0.18 \times 10^{-2}$ \\
\hline$\rho_{\text {tor }}=0.8$ & 11.32 & 13.51 & 8.84 & 0.76 & 1.85 & 2.06 & $0.08 \times 10^{-2}$ \\
\hline
\end{tabular}

Table I. Mean experimental parameters at $t=0.96 \mathrm{~s}$, at the selected radii.

\section{QUASILINEAR MODEL FOR THE EVALUATION OF FLUXES}

The results of the following section IV are based on the adoption of a quasilinear model, which allows to efficiently estimate ratios of fluxes starting from the outputs of linear flux-tube gyrokinetic simulations. This model in particular relies on a relation for the dependence of the saturated amplitude values of the electrostatic (ES) potential $\phi$ as a function of the poloidal wave number $k_{y}$. The particle and heat fluxes $F \equiv \Gamma, Q$ have been computed considering only the ES contribution (electromagnetic effects can be neglected since $\beta$ is small, as can be seen in Table I). The QL model includes the full ion scale $k_{y}$ spectra contribution, varying the number of linearly coupled $k_{x}$ Fourier modes for each $k_{y}$, starting from the fluxes which are obtained from the linear eigenmodes, 
similarly to $[6,18,20,21]$. Only the most unstable mode is considered at each wavenumber. The QL fluxes are given by the expression:

$$
F^{Q L}=A_{0} \sum_{k_{y}} w^{Q L}\left(k_{y}\right) F_{\text {norm }}^{L}\left(k_{y}\right) .
$$

Here, $A_{0}$ is a scaling factor associated to the absolute fluctuation amplitude, which does not need to be determined as we are interested in the zero particle flux condition, which can be obtained considering the ratio $\Gamma / Q . \quad F_{\text {norm }}^{L}\left(k_{y}\right)=$ $F^{L}\left(k_{y}\right) /\left|\hat{\phi}\left(k_{x}=0, k_{y}, z=0\right)\right|^{2}$ represents the $k_{y}$ spectral contribution to the flux which is evaluated with the fields (particle distribution, ES potential $\phi$ ) from the corresponding linear eigenmode, normalised with respect to $\left|\hat{\phi}\left(k_{x}=0, k_{y}, z=0\right)\right|^{2}$, where $\hat{\phi}\left(k_{x}, k_{y}, z\right)$ is the Fourier transform of the ES potential $\phi$ with respect to $\mathrm{x}$ and $\mathrm{y}$ and $z=0$ is the poloidal angle corresponding to the outer mid-plane. $F^{L}\left(k_{y}\right)$ are computed following [22] (see [8] for more details). The QL weights $w^{Q L}\left(k_{y}\right)$ specify the $k_{y}$ dependence of the relative saturation amplitude levels of the NL electrostatic potential. The following form is assumed for the QL weights for each $k_{y}$ :

$$
w^{Q L}=\left(\gamma /\left\langle k_{\perp}^{2}\right\rangle\right)^{\xi},
$$

which is usually referred to as the 'mixing length saturation rule' when $\xi=1$ [23]. Here, $\gamma$ is the growth rate of the considered linear mode and

$$
\left\langle k_{\perp}^{2}\right\rangle\left(k_{y}\right)=\frac{\sum_{k_{x}} \int k_{\perp}^{2}\left(k_{x}, k_{y}, z\right)\left|\hat{\phi}\left(k_{x}, k_{y}, z\right)\right|^{2} J(z) d z}{\sum_{k_{x}} \int\left|\hat{\phi}\left(k_{x}, k_{y}, z\right)\right|^{2} J(z) d z}
$$

is the flux-surface average of the squared perpendicular wave number, weighted by the mode amplitude $|\hat{\phi}|^{2}$, where $J(z)$ is the Jacobian associated to the curvilinear coordinate system $(x, y, z)$ along the flux-tube. Three choices of the number $n_{k x}^{Q L}$ of $k_{x}$ involved in the $\left\langle k_{\perp}^{2}\right\rangle$ computation have been considered: $n_{k x}^{Q L}=1\left(k_{x}=0\right), n_{k x}^{Q L}=3\left(k_{x}=-\Delta k_{x}, 0, \Delta k_{x}\right.$, following [21]), or $n_{k x}^{Q L}=n_{k x}\left(k_{x}=p \Delta k_{x}, p=-n_{k x} / 2+1,-n_{k x} / 2+\right.$ $\left.2, \ldots, n_{k x} / 2\right)$. It should thus be emphasised that $n_{k x}^{Q L}$ is in general distinct from the number $n_{k x}$ of $k_{x}$ values considered in the linear simulation. $n_{k x}^{Q L}$ is only related to the post processing of the simulation data when computing the QL fluxes. In our analysis, all the 9 combinations of $n_{k x}^{Q L}=1,3, n_{k x}$ and $\xi=1,2,3$ have been considered, choosing a posteriori the ones that best matched the NL results (see section IV B). To lighten the notation, the QL fluxes $F^{Q L}$ will be referred to as $F$ throughout the paper.

In the same spirit, an 'average' QL estimate $\omega^{Q L}$ of the most unstable mode frequency $\omega$ has been defined, similarly to [6], as a weighted sum over the poloidal wave number spectrum of the real frequency $\omega$, with weights equal to the QL ones given by Eq.(2), according to

$$
\omega^{Q L}=\sum_{k_{y}} \omega\left(k_{y}\right) w^{Q L}\left(k_{y}\right) / \sum_{k_{y}} w^{Q L}\left(k_{y}\right) .
$$

This value gives a quantitative measure of the type of mode most significantly contributing to the QL fluxes. It is positive in a dominant ITG regime, while it is negative in a dominant TEM regime, according to GENE conventions.

To correctly account for the contributions to the fluxes from a wide range of ion scales and to be consistent in the QL-NL comparison, the QL fluxes have been computed considering a $k_{y}$ spectrum of modes $k_{y}=p k_{\mathrm{y}, \min }, p=0,1, \ldots, n_{k y}$, with $k_{\mathrm{y}, \min } \rho_{s}=5 \cdot 10^{-2}$ (the same considered in the NL simulations), where $\rho_{s}=c_{s} / \Omega_{i}$ is the sound Larmor radius and $\Omega_{i}$ the ion cyclotron frequency. Typically, $n_{k y}=28$.

\section{RADIAL DEPENDENCE OF THE PEAKING FACTOR}

\section{A. Quasilinear analysis}

In order to start to characterise the turbulence at the different radii of analysis, linear $k_{y}$ scans have been performed at experimental mean parameters (see Table I), computing the growth rate and frequency spectra. The results are shown in Fig.2 (a) and (b), respectively, in physical units. Ion Temperature Gradient (ITG) and Trapped Electron Modes (TEM) turbulence regimes are identified by positive and negative frequencies respectively, according to GENE conventions. Except for $\rho_{\text {tor }}=0.5$, that stays in a TEM regime throughout its spectum, and neglecting the small ITG region at large scales for $\rho_{\text {tor }}=0.4$, which will be shown not to contribute to the particle flux, the turbulence moves from ITG to TEM with increasing $k_{y}$ at all radii. The minimum $k_{y}$ corresponding to ITG has been indicated by a square marker at each radius. Thus, since only the smaller wave numbers contribute significantly to the fluxes, the particle flux is mainly due to a dominantly TEM regime at all radii. This appears clearly in Fig.2 (c), where the QL estimates of the particle flux spectra at different radii are shown, indicating that it is non vanishing only in the TEM regime within the whole radial domain. For simplicity, only the results obtained with the QL model parameters $n_{k x}^{Q L}=3$ and $\xi=2$, which best matches the NL results in the present analysis (see section IV B and [8]), are shown here. It is worth noting that the maxima of $\Gamma / Q_{i}$ scale almost linearly with $\Gamma_{\mathrm{GB}} / Q_{\mathrm{GB}}=1 / T_{e}$, where $Q_{\mathrm{GB}}=\sqrt{m_{i}} n_{e} T_{e}^{5 / 2} / e^{2} R^{2} B_{0}^{2}$, since $T_{e}$ decreases from $0.76 \mathrm{keV}$ to $0.21 \mathrm{keV}$ with increasing radius from $\rho_{\text {tor }}=0.4$ to $\rho_{\text {tor }}=0.8$. Therefore, the $T_{e} \Gamma / Q_{i}$ maxima are similar at all radii, which is quite remarkable given the change of parameters in Table I.

Starting from the mean experimental values of the parameters, particle flux scans in the logarithmic density gradient have been performed at different radii to investigate the radial dependence of the $\mathrm{PF}=\mathrm{R} / \mathrm{L}_{\mathrm{n}}(\Gamma=0)$. For each value of $R / L_{n}$, 9 different QL models have been used to compute the particle flux (all the combinations of $\xi=1,2,3$ and $n_{k x}^{Q L}=1,3,48$ ), to estimate the sensitivity of the results on the QL model parameters. The difference between the maximum and minimum values of $T_{e} \Gamma / Q_{i}$, for each $R / L_{n}$, gives an error bar associated to the choice of the QL model. The results are shown in Fig.3 (a), with different colors corresponding to different radial positions. The QL error bar is represented by the shaded regions and only the curves corresponding to the 'best model' $\xi=2$, $n_{k x}^{Q L}=3$ are shown as solid lines. The curves intersect the $\Gamma=0$ line at the PF values. The square markers indicate the minimum $R / L_{n}$ with $\omega^{Q L}<0$, that is the approximate bound- 

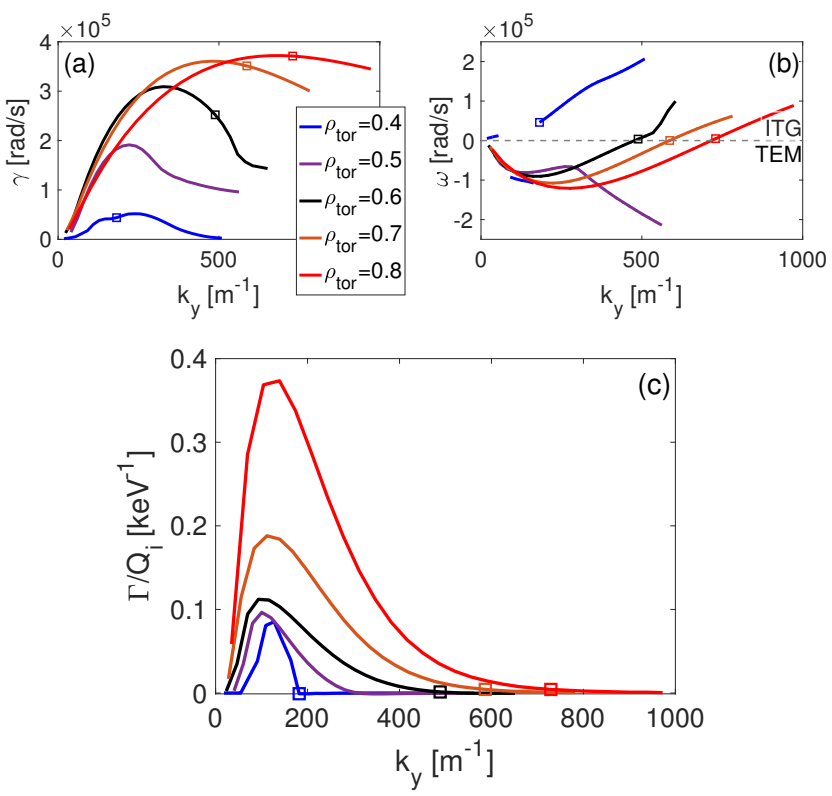

Figure 2. (color online) (a) and (b) growth rate and frequency spectra, respectively, varying radius from $\rho_{\text {tor }}=0.4$ to $\rho_{\text {tor }}=0.8$, with $k_{y} \rho_{s} \in[0.05,1.4]$. The square markers indicate the minimum $k_{y}$ with positive frequency (ITG); (c) QL estimates of the particle flux spectra, divided by the ion heat flux, for the same cases of (a) and (b). The QL model parameters are $n_{k x}^{Q L}=3$ and $\xi=2$.

ary between a dominantly ITG regime (at lower $R / L_{n}$ ) and a dominantly TEM one (at higher $R / L_{n}$ ). This means that the $\mathrm{PF}$ is obtained close to the ITG-TEM threshold at all radii.

The PF radial dependence is summarised in Fig.3 (b), where PF is represented vs $\rho_{\text {tor }}$, following the same color code of (a). The error bars are estimated by the difference of the maximum and minimum PF obtained varying the 9 QL models. The QL estimate of the PF varies with radius by $\sim 40 \%$ in the stiff region $0.4 \lesssim \rho_{\text {tor }} \lesssim 0.8$, remaining almost constant for $\rho_{\text {tor }}>0.6$. These values seem to considerably underestimate the exp. ones (see the green line and Table I).

Attempting to explain this discrepancy at the larger radii, the dependence of the PF on the ion and electron temperature gradients $R / L_{T i, e}$ has been investigated considering the three radial positions $\rho_{\text {tor }}=0.4,0.6,0.8$, individually varying $R / L_{T i, e}$ by $\pm 20 \%$ around the mean experimental values. For each value of $R / L_{T i, e}$, the PF has been obtained performing a particle flux $R / L_{n}$ scan. The results of the PF $R / L_{T i}$ and $R / L_{T e}$ scans are shown in Fig.4 (a) and (b) respectively. They indicate that the dependence of the PF on the temperature gradients is stronger but still moderate at the smallest radius $\rho_{\text {tor }}=0.4$, while it becomes even smaller at the larger radii, where the variations of the $\mathrm{PF}$ with varying $R / L_{T i, e}$ are very similar to each other at $\rho_{\text {tor }}=0.6$ and $\rho_{\text {tor }}=0.8$. Overall, the PF slightly increases with increasing $R / L_{T i}$ while it does not vary significantly with increasing $R / L_{T e}$. This behaviour is consistent with the small dependence of the particle flux on $R / L_{T e}$ which had been found in [24] (see Fig.5) and the small thermodiffusive contribution to the electron particle flux as compared to the corresponding contribution to the ion par-
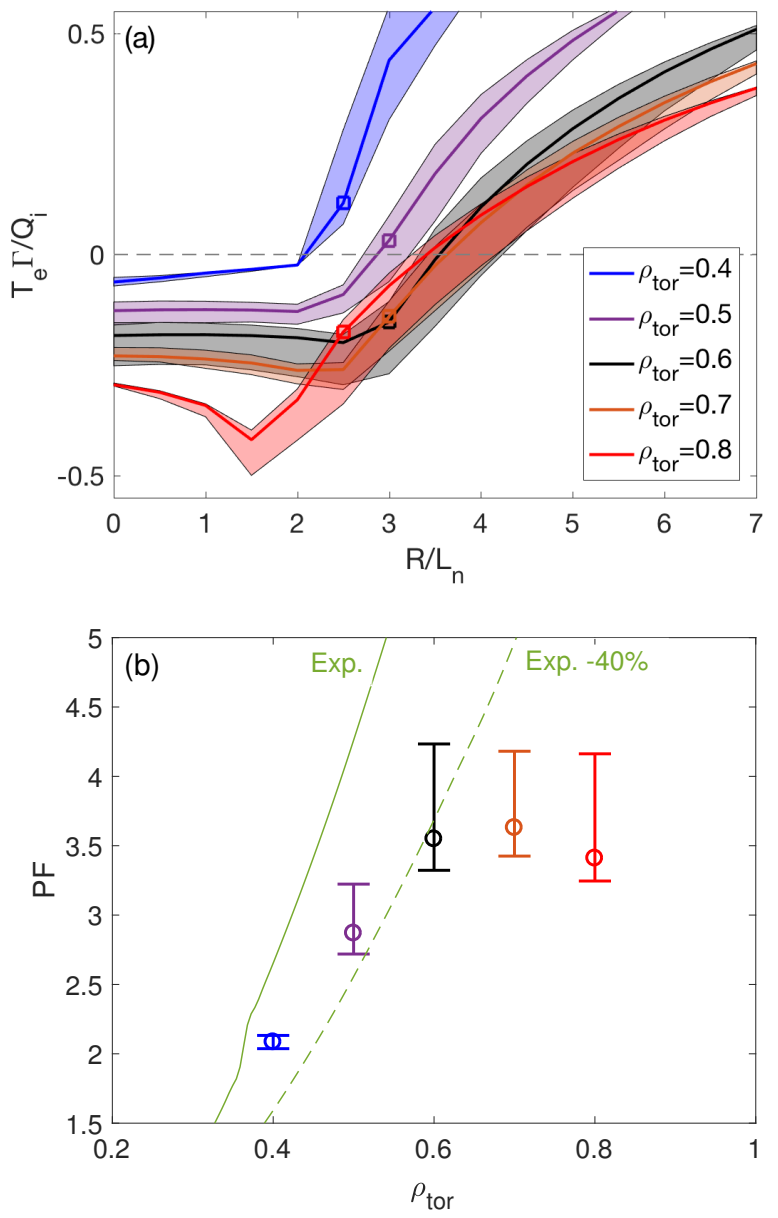

Figure 3. (color online) (a) QL $T_{e} \Gamma / Q_{i} R / L_{n}$ scan. The five different radii of analysis $\rho_{\text {tor }}=0.4,0.5,0.6,0.7,0.8$ are indicated by different colors. The thick solid lines indicate the results of the $n_{k x}^{Q L}=3, \xi=2$ QL model ('best model'). The shaded regions represent the error bar associated to the extremal QL results varying $n_{k x}^{Q L}$ and $\xi$. The square markers are placed at the smallest $R / L_{n}$ so that $\omega^{Q L}<0$, that is the smallest $R / L_{n}$ compatible with a TEM dominated regime. (b) PF radial scan. The circle markers indicate the QL estimates with the 'best model', while the error bars correspond to the intersections of Fig. (a) shaded regions with the $\Gamma=0$ line. Finally, the experimental values of $R / L_{n}$ are shown as a solid green line, while the same values minus $40 \%$ are indicated by a green dashed line.

ticle flux that has been observed in [8] (see Fig.3 (c) and (d)), both local analyses performed at $\rho_{\text {tor }}=0.6$. The weak dependence of the PF on $R / L_{T e}$ could be due to the fact that the PF is obtained as a balance of different contributions coming from ITG and mainly $R / L_{n}$-driven TEM. For more $R / L_{T e}$-driven TEM contributions one would expect a stronger dependence of the PF on $R / L_{T e}$. The largest PF variation which has been observed in this analysis is $\Delta\left(R / L_{n}\right)_{\max } \sim 1.4$ within the $R / L_{T i}$ scan at $\rho_{\text {tor }}=0.4$, while the smallest one is $\Delta\left(R / L_{n}\right)_{\min } \sim 0.2$ whithin the $R / L_{T e}$ scan at $\rho_{\text {tor }}=0.6$.

Since the PF has been found not to vary considerably with varying $R / L_{T i}$ and $R / L_{T e}$ at the larger radii, the experimental error bars on the ion and electron temperature gradients can 

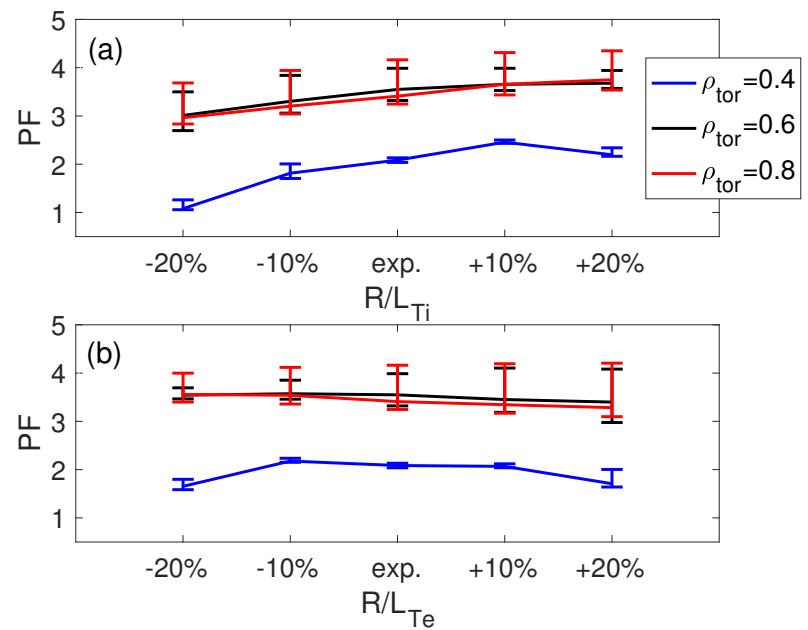

Figure 4. (color online) Dependence of PF on temperature gradient $R / L_{T}$ of (a) ions and (b) electrons. Results obtained with the QL model. Gradient values are varied within $\pm 20 \%$ of mean experimental values. The three radii $\rho_{\text {tor }}=0.4,0.6,0.8$ are indicated by different colors, following the same color code of Fig.3. The thick solid lines indicate the results of the $n_{k x}^{Q L}=3, \xi=2 \mathrm{QL}$ model ('best model'), while the error bars show the extremal QL results varying $n_{k x}^{Q L}$ and $\xi$, consistently with Fig.3.

not be invoked to explain the discrepancy between the simulated PF and the experimental one. One should nonetheless not forget that the present analysis is neglecting collisions and impurities, as well as global effects, therefore this result is not surprising. Nevertheless, letting aside the two outer radii, where the large experimental values are clearly not recovered, the other PF values are all within the wide experimental error bars $( \pm 40 \%)$. If this picture will persist in a future analysis including collisions and other effects, the fit of the experimental $n_{e}$ in the $\rho_{\text {tor }}>0.6$ region should be repeated in order to get the best possible agreement with the PF results based on imposing the zero particle flux constraint. This is exactly the purpose of the present approach, imposing physical constraints such as the zero particle flux, in order to reduce the experimental uncertainties.

\section{B. Validation of the quasilinear results by means of nonlinear simulations}

Nonlinear gyrokinetic simulations have been run in a subset of cases in order to test the validity of the QL results. The two radii $\rho_{\text {tor }}=0.4,0.8$ have been considered $\left(\rho_{\text {tor }}=0.6\right.$ has been extensively analysed in [8]), choosing three $R / L_{n}$ values for each radius. The results are shown in Fig.5, where the NL particle fluxes are compared with the QL ones, obtained varying the QL model parameters. The NL results are indicated by square black markers and referred to as NLsj and NLlj, with $\mathrm{j}=1,2,3$, corresponding to $\rho_{\text {tor }}=0.4$ and $\rho_{\text {tor }}=0.8$ respectively, where 's' and ' 1 ' in NLsj and NLlj stand for 'small radius' and 'large radius' respectively, while the QL results are shown as colored lines. The NL and QL estimates of the $\mathrm{PF}$ are found to be in a very good agreement for all the considered cases, thus validating the adopted QL model in the considered parameters range.
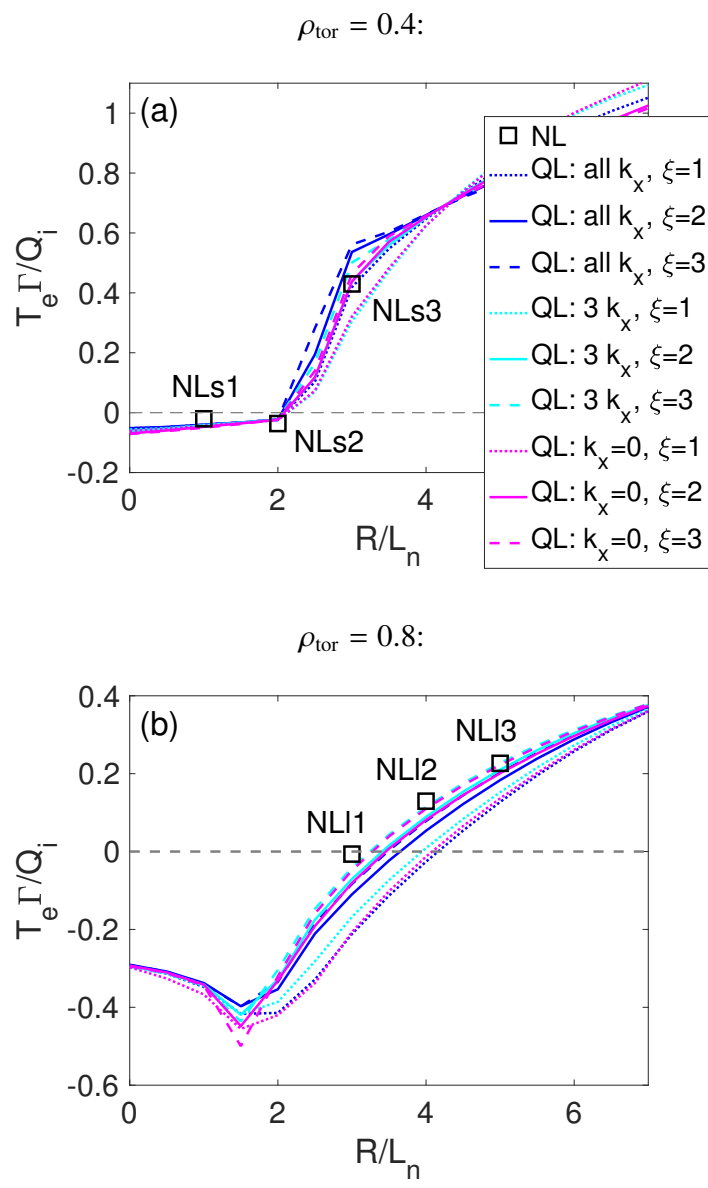

Figure 5. (color online) (a) and (b) comparison between QL and NL estimates of $T_{e} \Gamma / Q_{i}$, represented versus $R / L_{n}$, at $\rho_{\text {tor }}=0.4$ and $\rho_{\text {tor }}=0.8$ respectively. The QL results with the 9 combinations of $n_{k x}^{Q L}=1,3,28$ and $\xi=1,2,3$, shown as colored lines, are confronted with the NL results at selected values of $R / L_{n}$, indicated by square markers.

In order to further verify the reliability of the QL results, the NL spectra have been compared with the QL ones. Fig.6 shows this comparison for the two cases NLs3 and NL13. The NL results are shown as thick black lines, while the QL results with best QL-NL agreement for which $n_{k x}^{Q L}=3$ and $\xi=2,3$ are shown in cyan. The grey shaded region shows the associated QL error bar, ranging from the minimum to the maximum QL value for each $k_{y}$, varying the QL model parameters. The NL-QL spectral agreement is also very good, in particular considering NL13 case, further validating the QL analysis.

\section{GLOBAL EFFECTS ON PEAKING FACTOR}

With the aim of investigating the global effects on the PF, the $\mathrm{PF} \sim 3$ result which has already been obtained in the local $\mathrm{NL}$ analysis [8] has been compared with a global simulation result. We started by considering two NL flux-tube simulations from the local analysis at $\rho_{\text {tor }}=0.6$. The first, which has been run with $R / L_{n}=3$ and is referred to as NL2 in 

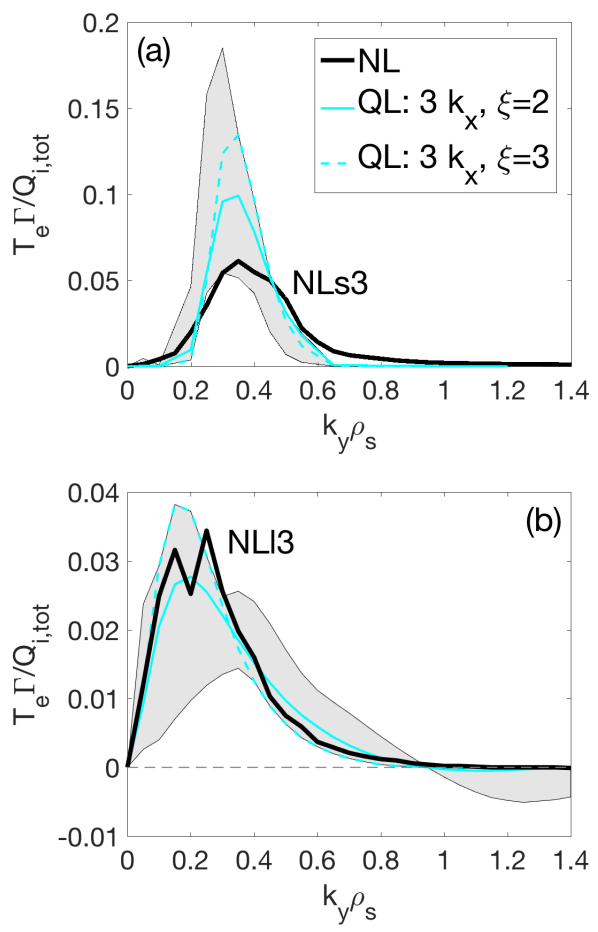

Figure 6. (color online) (a) and (b) Comparison of QL and NL particle flux $k_{y}$ spectra, in $Q_{i, t o t} / T_{e}$ units, where $Q_{i, t o t}=\sum_{k y} Q_{i}\left(k_{y}\right)$, for the NLs3 and NL13 cases from Fig.5 (a) and (b) respectively. The QL results with $n_{k x}^{Q L}=3$ and $\xi=2,3$ are represented as cyan solid and dashed lines respectively, while NL results are in black. The shaded regions indicate the QL error bar.

[8], has almost vanishing output particle flux (see Fig.15 (a) in [8]), and therefore essentially corresponds to the local PF. The second, run with $R / L_{n}=5$ and referred to as NL3 in [8], displays outward particle flux. The two corresponding output particle fluxes are shown in Fig.7, in gyro-Bohm units, with $\Gamma[G B]=\Gamma / \Gamma_{\mathrm{GB}}$.

Local reference NL simulations at $\rho_{\text {tor }}=0.6$ :

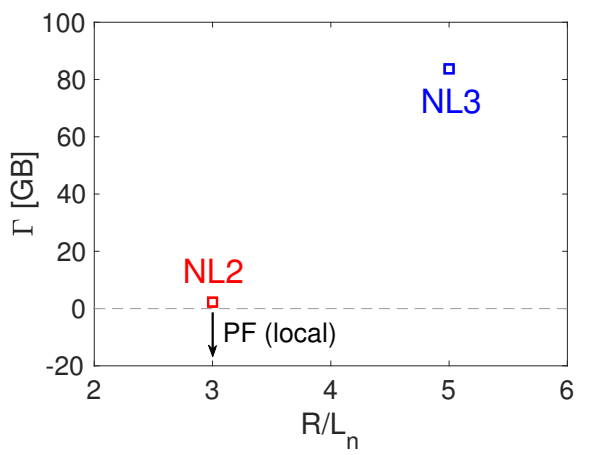

Figure 7. Particle fluxes, in GB units, corresponding to the two local flux-tube simulations NL2 and NL3 at $\rho_{\text {tor }}=0.6$, corresponding to $R / L_{n}=3,5$ respectively (for details and notations see [8]). The NL2 simulation almost satisfies the zero particle flux condition, therefore $R / L_{n} \sim 3$ is considered as the local estimate of the PF.

Two NL global GD simulations, which correspond to NL2 and NL3 respectively, have been run. Their output particle fluxes have been found a posteriori to be inward and outward respectively, so that a global PF radial profile has been estimated interpolating their $R / L_{n}$ radial profiles with respect to $\Gamma$ at $\Gamma=0$. More details are provided in the following.

\section{A. Simulations settings}

Given the numerical cost of such global simulations, only the radial region $0.35<\rho_{\text {tor }}<0.85$ has been simulated. Analytic density and temperature profiles with constant logarithmic gradients in the stiff region $0.4 \lesssim \rho_{\text {tor }} \lesssim 0.8$ have been used, following [16, 25], matching the experimental parameters at $\rho_{\text {tor }}=0.6$, the radius which had been chosen in [8] to perform the local analysis, in order to compare the global results with the local ones at that position. The analytical form of the density profiles is

$$
\frac{n\left(\rho_{\text {tor }}\right)}{n\left(\rho_{\text {tor }, \mathrm{c}}\right)}=\left[\frac{\cosh \left(\frac{\left(\rho_{\text {tor }}-\rho_{\text {tor, },}\right)+\delta n}{\Delta n}\right)}{\cosh \left(\frac{\left.\rho_{\text {tor }}-\rho_{\text {tor }, \mathrm{c}}\right)-\delta n}{\Delta n}\right)}\right]^{-\kappa_{n} \epsilon \Delta n / 2},
$$

where $\rho_{\mathrm{tor}, \mathrm{c}}=0.6$ is the toroidal radius at the center of the considered radial box, which has been set equal to the local analysis radius. $\delta n=0.2$ and $\Delta n=0.02$ set the gradient profile width and decay length respectively (see Fig.8 (b)), while $\kappa_{n}=\max \left(R / L_{n}\right)$ denotes the maximum density gradient. Furthermore, $\epsilon=\alpha / R$ is the inverse aspect ratio at the last close flux surface. The temperature profiles have the same analytical form, with corresponding parameters $\delta T=\delta n, \Delta T=\Delta n$ and $\kappa_{T}=\max \left(R / L_{T}\right)$. The density and temperature profiles, with the corresponding logarithmic gradients, are shown in Fig.7 (a) and (b) respectively. The radial simulation domain is centered at the local analysis radius. $n_{x}=200$ radial points have been considered over the $L_{x}=74 \rho_{s}$ domain, corresponding to approximately 2.7 points for each ion sound Larmor radius. The box sizes $L_{\mathrm{v} \|}, L_{\mu}$ and the resolutions $n_{\mathrm{v} \|}, n_{\mu}$ in the velocity space $\left(\mathrm{v}_{\|}, \mu\right)$ have been adapted in order to have a sufficiently large box at the inner radial boundary (corresponding to the maximum temperature) and sufficient resolution at the outer radial boundary (corresponding to the minumum temperature), obtaining $L_{\mathrm{v} \|} / \mathrm{v}_{\mathrm{T}, \mathrm{j}}=4, \mathrm{~L}_{\mu} \mathrm{B}_{0} / \mathrm{T}_{\mathrm{j}}=14$ and $n_{\mathrm{v} \|}=72, n_{\mu}=36$, where $T_{j}$ and $\mathrm{v}_{\mathrm{T}, \mathrm{j}}$ are the temperature and the thermal velocity of the $\mathrm{j}$-th species, respectively, at the center of the radial simulation box. $n_{z}=32$ points have been considered in the parallel direction. The minimum poloidal wavenumber $k_{\mathrm{y}, \min } \rho_{s} \sim 0.05$, corresponding to the toroidal number $n=4$, has been chosen to compare with the $\mathrm{NL} / \mathrm{QL}$ local results that have been obtained considering as well $k_{\mathrm{y}, \min } \rho_{s}=0.05$.

Despite the fact that in the local NL simulations only fluctuations with $k_{y} \rho_{s} \lesssim 1$ significantly contribute to the fluxes, a larger maximum poloidal wave number $k_{y, \max } \rho_{s} \sim 1.7$ has been set in the global simulations, allowing smaller scales to possibly contribute in the latter case. This corresponds to simulating $n_{k y}=32$ poloidal modes, which was compatible with the available computational resources. This choice turned out to be conservative, since only $k_{y} \rho_{s} \lesssim 1$ contribute 

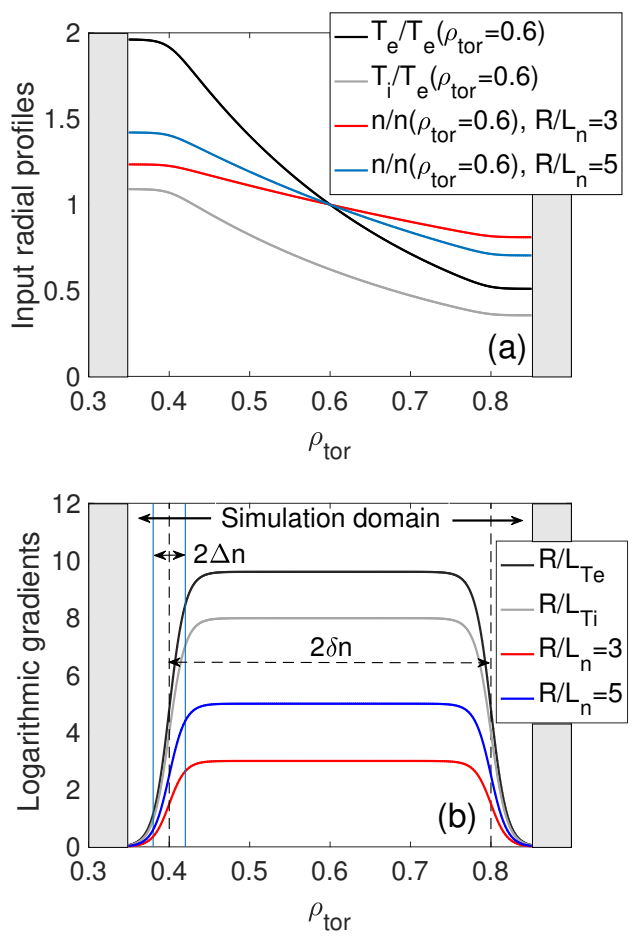

Figure 8. (color online) (a) Input density and temperature profiles of the two NL global simulations with $R / L_{n}=3,5$ respectively. (b) Logarithmic gradients of the profiles shown in (a).

to the fluxes even in the global simulations, as will be shown. So to summarise, the NL simulation grid has been set up to $n_{x} \times n_{k y} \times n_{z} \times n_{\mathrm{v} \|} \times n_{\mu}=200 \times 32 \times 32 \times 72 \times 36$. Dirichlet boundary conditions have been assumed in the radial coordinate. Since fluctuations should be small close to the boundaries for consistency with the selected boundary conditions, Krook-type operators $-v_{\text {Krook,in }} \delta f$ and $-v_{\text {Krook,out }} \delta f$ have been used at the inner and outer boundary respectively, where $\delta f$ is the perturbation of the considered distribution function and

$$
\begin{aligned}
v_{\text {Krook,in }}\left(\rho_{\text {tor }}\right) & =2 \frac{c_{s}}{R}\left(\frac{\rho_{\text {tor }}-0.1}{0.1}\right)^{4} \chi_{[0,0.1]}\left(\rho_{\text {tor }}\right), \\
v_{\text {Krook }, \text { out }}\left(\rho_{\text {tor }}\right) & =2 \frac{c_{s}}{R}\left(\frac{\rho_{\text {tor }}-0.9}{0.1}\right)^{4} \chi_{[0.9,1]}\left(\rho_{\text {tor }}\right),
\end{aligned}
$$

are polynomial functions of the radius which are non-zero inside the inner $\left(0<\rho_{\text {tor }}<0.1\right)$ and outer $\left(0.9<\rho_{\text {tor }}<1\right)$ buffer zones respectively ( $10 \%$ of the radial domain on each side), increasing towards the boundaries up to the values $v_{\text {inner }}=v_{\text {outer }}=2 c_{s} / R$, approximately two times the corresponding maximum linear growth rate. $\chi_{I}$ indicates the characteristic function of the interval $I$. Krook-type heat and particle sources have been finally adopted in order to keep temperature and density profiles fixed on average, allowing the GD simulations. The amplitudes of the Krook-type heat and particle source have been set to $\gamma_{H} R / c_{s}=0.2$ and $\gamma_{P} R / c_{s}=0.1$ respectively, equal to approximately $1 / 10$ of the maximum linear growth rate, significantly smaller than the linear growth rates in order to affect only the long-time dynamics. Nevertheless, a study of the robustness of our results with respect to varying the Krook-type heat and particle source is presented in Appendix A, showing the robustness of our analysis. We refer to [25-28] for further details on buffer regions and sources implementation in GENE. In order to speed up the simulations and be able to obtain the results within our computational resources, we assumed the 'heavy electrons' approximation $\left(m_{i} / m_{e}=400\right)$. It has been verified, repeating NL3 local flux-tube simulation at $\rho_{\text {tor }}=0.6$, that this assumption modifies $\Gamma$ by only $\sim 2 \%$, making this approximation acceptable in our case. The simulations have been run up to at least $t \sim 80 R / c_{s}$ to collect enough statistics.

\section{B. Global results}

The NL global particle fluxes, compared with the local results at $\rho_{\text {tor }}=0.6$, are shown in Fig.9 (a). The two particle flux radial profiles, obtained from the output of the global simulations with $R / L_{n}=3$ and $R / L_{n}=5$ are illustrated by red and blue lines respectively. A radial-dependent error bar is provided, equal to the standard deviation of the running average, computed over the last $\Delta t \sim 10 R / c_{s}\left(\rho_{\text {tor }}=0.6\right)$ of the simulations. The corresponding local flux-tube values are indicated by red and blue square markers respectively. It has to be noted that both global and local fluxes are given in gyro-Bohm units, with gyro-Bohm normalisation corresponding to $\rho_{\text {tor }}=0.6$.

The local particle fluxes overestimate the global profile for both simulations. In particular, the NL3 local simulation particle flux considerably overestimates the corresponding global result. This behaviour was expected, from previous studies $[17,18]$, since $\rho^{\star}=\rho_{s} / \alpha \sim 1 / 150$ at $\rho_{\text {tor }}=0.6$ is sufficiently large to produce noticeable finite Larmor radius effects on the fluxes.

These results have been used to obtain the global PF profile. With this aim, the $R / L_{n}$ profiles have been linearly interpolated versus their corresponding particle fluxes, at $\Gamma=0$. The result is shown in Fig.9 (b). The global PF radial profile, represented by a thick black line, is locally close to the fluxtube value at $\rho_{\text {tor }}=0.6$, indicated by a square black marker. Therefore, the global results on the PF turn out to be relatively weak.

The error on this simple linear interpolation procedure is expected to be small, since the simulation with $R / L_{n}=3$ is already very close to the zero particle flux condition at all radii. The relative error which is induced by the error bars in Fig. 9 (a) is of order $\sim 1 / 1000$, since $\Gamma$ [GB] versus $R / L_{n}$ is steeply crossing $\Gamma=0$ at the PF at each radius, thus it is negligible. The input density gradient profiles (see Figures 8 (b) and 9 (b)) have been used in the interpolation procedure, since the output density gradient profiles were radially oscillating due to profile corrugation (see Appendix B). The PF which has been obtained using the effective output gradient profiles instead of the input ones is shown as a dotted magenta line in Fig.9 (b), for completeness. To smooth out its radial oscillations, a linear fit has been considered on the central radial region (thick solid magenta line), which is in very good agreement with the PF that has been computed using the input gradients, validating the adopted procedure.

The radially averaged particle fluxes corresponding to the two simulations with $R / L_{n}=3,5$ have been compared in 

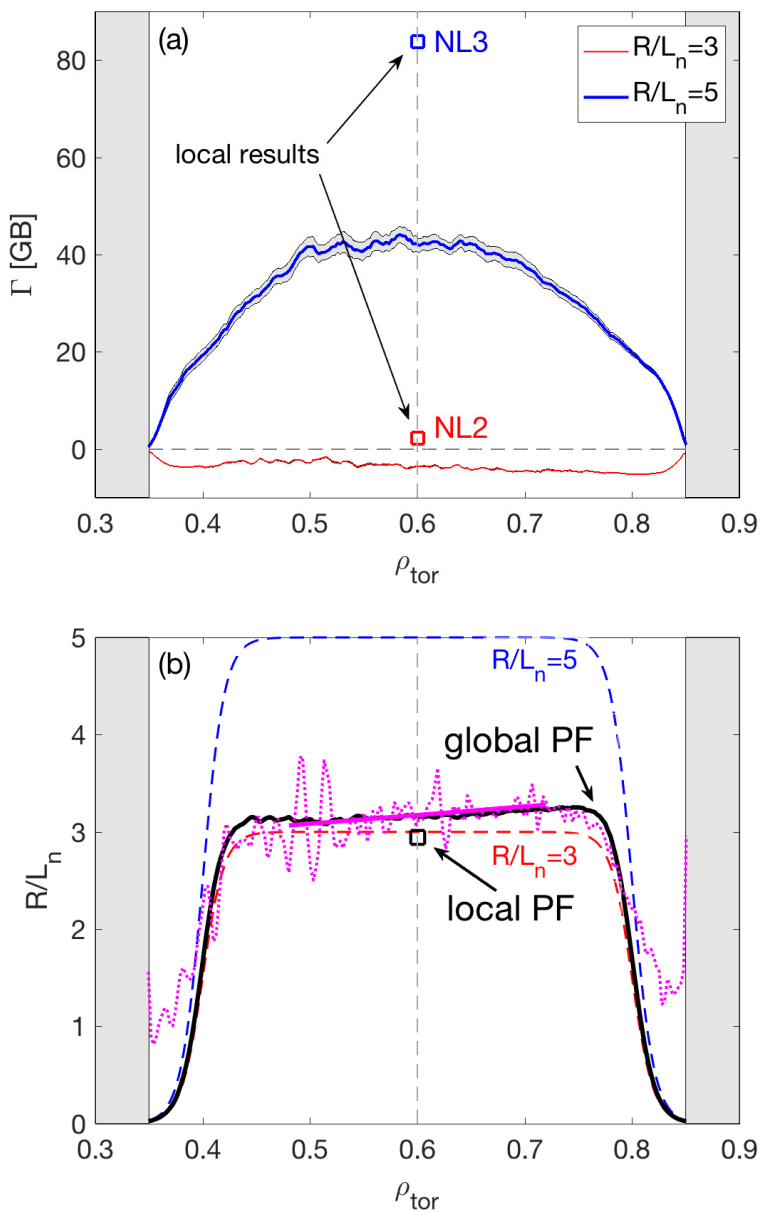

Figure 9. (color online) (a) Global particle fluxes in GB units, corresponding to the two simulations with $R / L_{n}=3,5$ respectively. The error bars are estimated at each radius by \pm the standard deviation of the running average over the last $\Delta t=10 R / c_{s}$. The averages have been performed on the time intervals $t \sim[60,120] R / c_{s}$ and $t \sim[50,80] R / c_{s}$ for the simulations corresponding to $R / L_{n}=3$ and $R / L_{n}=5$ respectively. The square markers indicate the local flux tube values at $\rho_{\text {tor }}=0.6$. (b) Global PF profile (black) computed by linearly interpolating the input $R / L_{n}$ profiles (red and blue) wrt. the corresponding particle flux profile $\Gamma\left(\rho_{\text {tor }}\right)$ (already shown in (a): red and blue) at $\Gamma=0$. The result is compared with the PF that is estimated using the final $R / L_{n}$ profiles from the simulation output, averaged on the same $t \sim[60,120] R / c_{s}$ and $t \sim[50,80] R / c_{s}$ time intervals that have been used to produce (a) (cyan $R / L_{n}$ profiles in Fig.12 (b), for the $R / L_{n}=5$ case), instead of the input $R / L_{n}$ profiles. This PF estimate is shown as a dotted magenta line, together with its linear fit over the $0.48<\rho_{\text {tor }}<0.72$ central region (thick magenta line). Finally, the local value of the PF is shown by a square black marker, for comparison.

more detail with the flux-tube values at $\rho_{\text {tor }}=0.6$. The results, shown in Fig.10 indicate that the $T_{e}\left(\rho_{\text {tor }}=0.6\right) \Gamma / Q_{i, t o t}$ spectrum, where $Q_{i, t o t}=\sum_{k y} Q_{i}\left(k_{y}\right)$, is in very good agreement with the local one for the $R / L_{n}=5$ case (Fig.10 (b)), that is far from the zero particle flux condition. This result may be surprising at first sight given the results shown in Fig.9 (a). Indeed, the absolute global particle flux in GB units is consider-
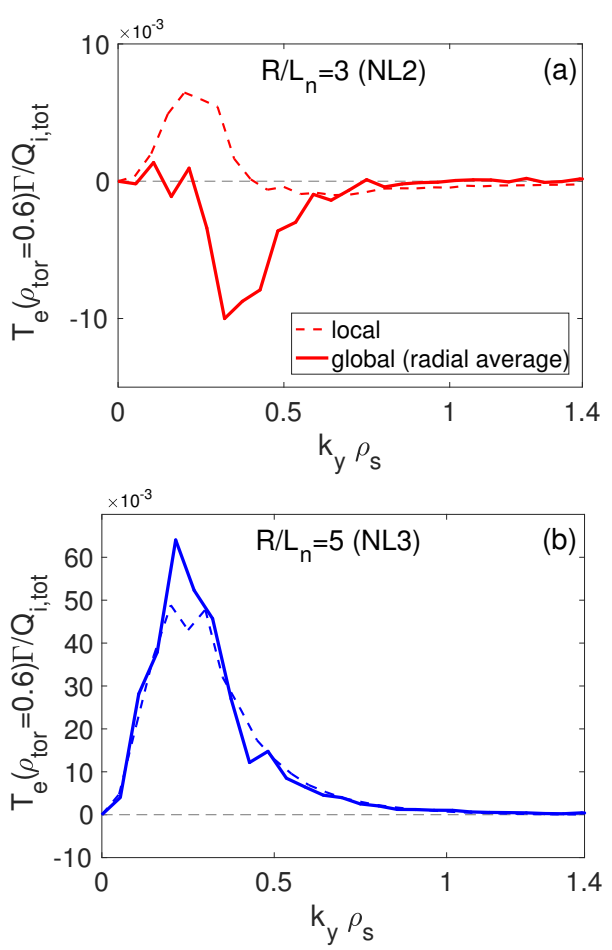

Figure 10. (color online) (a) and (b) Radially averaged $k_{y}$ spectra, corresponding to the two global Nl simulations with $R / L_{n}=3,5$ respectively (solid), compared with the local spectra at $\rho_{\text {tor }}=0.6$ (dashed). Here, the ratio $T_{e}\left(\rho_{\text {tor }}=0.6\right) \Gamma / Q_{i}$ is shown, where for the global simulations, both $\Gamma$ and $Q_{i}$ are radially averaged on the whole radial box.

ably smaller than the local value at $\rho_{\text {tor }}=0.6$ at all radii. This led us to investigate the impact of global effects on the $\Gamma / Q_{i}$ flux ratio. This analysis, presented in Appendix B, shows that this flux ratio does not show significant global effects. The investigation of the generality of this remarkable property has still to be pursued, and is beyond the aim of this paper.

Finally, the global PF profile has been tested to be consistent with the zero particle flux condition. To this end, the PF profile has been radially integrated, keeping the local density at the center of the radial box fixed to its mean experimental value. The so obtained density profile (dotted black line in Fig.11 (b)) has been used as input to a third global simulation.

The output $\Gamma[G B]$ is presented as a dotted black line in Fig.11 (c). This profile is still non-zero, therefore it is not exactly matching the zero particle flux condition. This was expected, since the $\mathrm{PF}$ has been computed with a simple linear interpolation of $R / L_{n}$ wrt. $\Gamma$. Nevertheless, it is relatively close to zero (compared with the flux corresponding to the $R / L_{n}=5$ simulation (blue line in Fig.9).

This procedure has been iterated, first computing a new PF using this new $\Gamma[G B]$ (with the corresponding $R / L_{n}$ profile) together with the $R / L_{n}=3$ data to compute $R / L_{n}(\Gamma=0)$ and then integrating it to produce a new input density profile for a global simulation (solid black line in Fig.11 (b)). The final output $\Gamma[G B]$ is shown as a solid black line in Fig.11 (c). Eventually, the last $\Gamma[G B]$ profile has been used to compute a PF profile (solid black line in Fig.11 (a)). During this it- 

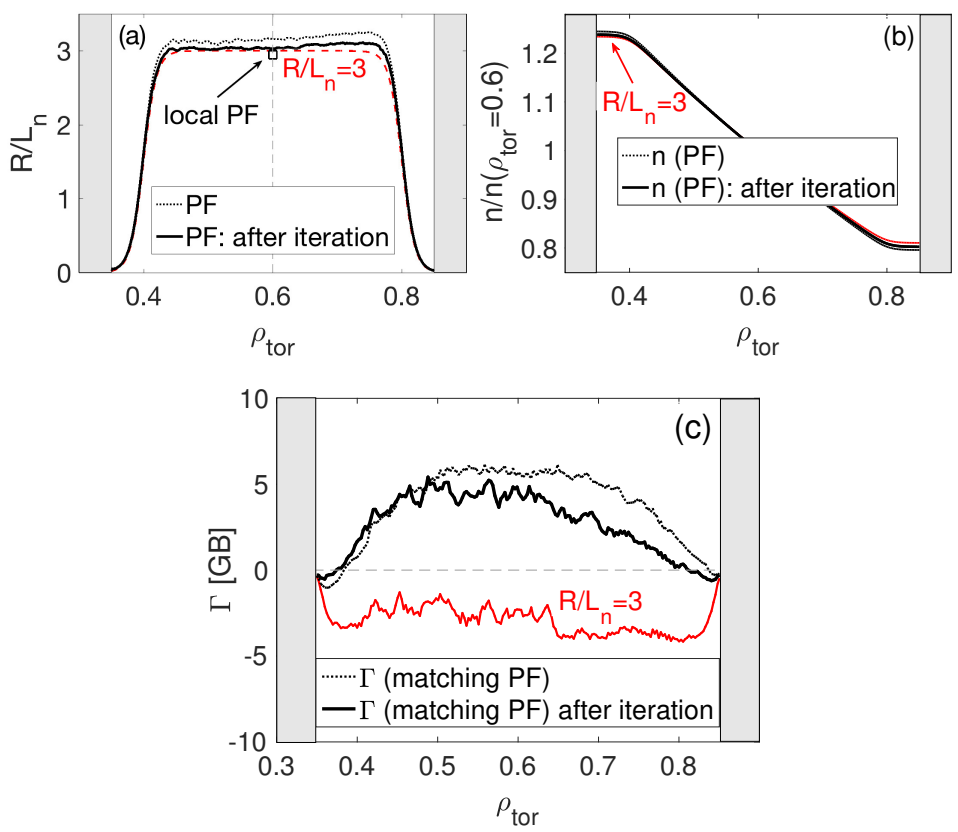

Figure 11. (color online) Iterative process to refine the PF evaluation and check the zero particle flux condition. (a) Global PF (dotted, same as Fig.9 (b)) and global PF after one iteration of the process (solid). (b) Density profiles which are obtained radially integrating the ones in (a), used as inputs for the global simulations in the iterative process. (c) Output particle fluxes in GB units, corresponding to input density profiles from (b).

erative procedure, the global PF becomes closer to the local value at $\rho_{\text {tor }}=0.6$, even more confirming that the global effects on the PF are negligible in our case. The final $\Gamma[G B]$ profile (solid black line in Fig.11 (c)) is still non-zero. Nevertheless, since the final $\Gamma[G B]$ is positive and the original $\Gamma[G B]$ matching $R / L_{n}=3$ (red line in Fig.11 (c)) was negative, the PF should stay between the red and black lines of Fig.11 (a), which are already very close. Moreover, the $\Gamma$ profiles have error bars resulting from insufficient statistics/not fully relaxed simulations, therefore there is a limit to such iterations further improving the PF estimate. Therefore, after this iterative analysis, the error bar on our estimate of the PF results to be small. These results further reflect the stiffness of the profiles. A small variation of the gradients (Fig.11 (a)) leads to a significant change of the fluxes (Fig.11 (c)).

\section{CONCLUSIONS}

A detailed analysis of the radial dependence of the PF, that is the density gradient corresponding to zero particle flux, together with an investigation of the global effects that could affect its estimate, has been performed running GENE gyrokinetic simulations, considering a TCV discharge of interest for momentum transport study. This shot has been previously analysed by the authors at fixed radial position, neglecting global effects [8]. The PF radial dependence has been evaluated adopting a QL model for the evaluation of the particle flux in section IV, starting from linear gyrokinetic re- sults, showing that it varies by $\sim 40 \%$ in an inner radial region $0.4<\rho_{\text {tor }}<0.6$, consistently with experimental $R / L_{n}$, while it stays constant for $0.6<\rho_{\text {tor }}<0.8$, contrarily to the experimental $R / L_{n}$ which continues to increase with increasing radius. The PF dependence on the electron and ion temperature gradients has been eventually estimated, considering a $\pm 20 \%$ variation of $R / L_{T i, e}$ around the mean experimental values at the three radii $\rho_{\text {tor }}=0.4,0.6,0.8$. The PF slightly increases at all radii with increasing $R / L_{T i}$, while it does not vary significantly with varying $R / L_{T e}$. QL results have been validated comparing them with NL ones in a subset of cases (section IV B). QL-NL agreement has been found to be very good. Realistic geometry and kinetic electrons have been considered throughout the work. Collisions and impurities have been neglected for simplicity in the present analysis. These restrictions will be removed in a future work. Comparing to the present work will be useful to single out their effects.

In section $\mathrm{V}$, the global effects on the PF evaluation at $\rho_{\text {tor }}=0.6$ have been estimated, running two global gradientdriven NL gyrokinetic simulations, corresponding to output particle fluxes profiles of opposite sign. These outputs were used to compute the density gradient of zero particle flux at each radius. The results have been compared with the local flux-tube estimate of the PF, which had already been computed by means of NL flux-tube gyrokinetic simulations in [8]. The global effects on the PF ended up to be nearly negligible. Nonetheless, flux levels present finite $\rho^{*}$ effects (see Fig.9 (a) for $R / L_{n}=5$ ). Only the radial region $0.35<\rho_{\text {tor }}<0.85$ has been simulated and some simplifications had to be assumed in this global analysis, due to the computational cost of such simulations. Analytic input density and temperature profiles, with constant logarithmic gradients have been adopted, and a higher mass ratio $m_{i} / m_{e}=400$ has been set. While the latter approximation has been verified not to significantly impact the results, the former will have to be removed in a future work, to be consistent with the first part of the paper, where the PF has been found to vary for $\rho_{\text {tor }}<0.6$, when considering full experimental density and temperature profiles. This extension of the present work, together with other possible ones including taking into account collisions and impurities, would contribute to better compare the results with the experiment.

Finally, during the analysis, two remarkable effects have been observed, which are presented in the two appendices respectively. They are both related to considering the flux ratio $\Gamma / Q_{i}$ instead of the particle flux alone. The first effect consists in the stability of $\Gamma / Q_{i}$ with respect to the variation of the amplitude of the Krook-type sources that are adopted to keep the profiles fixed on average. The second one is the absence of global effects on $\Gamma / Q_{i}$. These behaviours hold in our particular case. If they would persist in a broader parameters range, they could be applied in general to density peaking studies.

\section{ACKNOWLEDGEMENTS}

This work has been carried out within the framework of the EUROfusion Consortium and has received funding from the Euratom research and training programme 2014-2018 under grant agreement number 633053. The views and opinions expressed herein do not necessarily reflect those of the 
European Commission. This work was supported in part by the Swiss National Science Foundation. The numerical simulations have been carried out on the MARCONI cluster at CINECA.

\section{Appendix A: Dependence of the global results on the amplitude of the Krook-type fluxes}

In order to test the robustness of our results, we varied the relaxation rates $\gamma_{H}$ and $\gamma_{P}$ of the Krook-type heat and particle sources, multiplying them by a factor 3 in case of profiles with $R / L_{n}=5$. The outcome of this numerical experiment is shown in Fig.12, where the results obtained with setting $\gamma_{H} R / c_{s}=0.6$ and $\gamma_{P} R / c_{s}=0.3$ are compared with the ones corresponding to $\gamma_{H} R / c_{S}=0.2$ and $\gamma_{P} R / c_{s}=0.1$ (section V B. Fig.12).
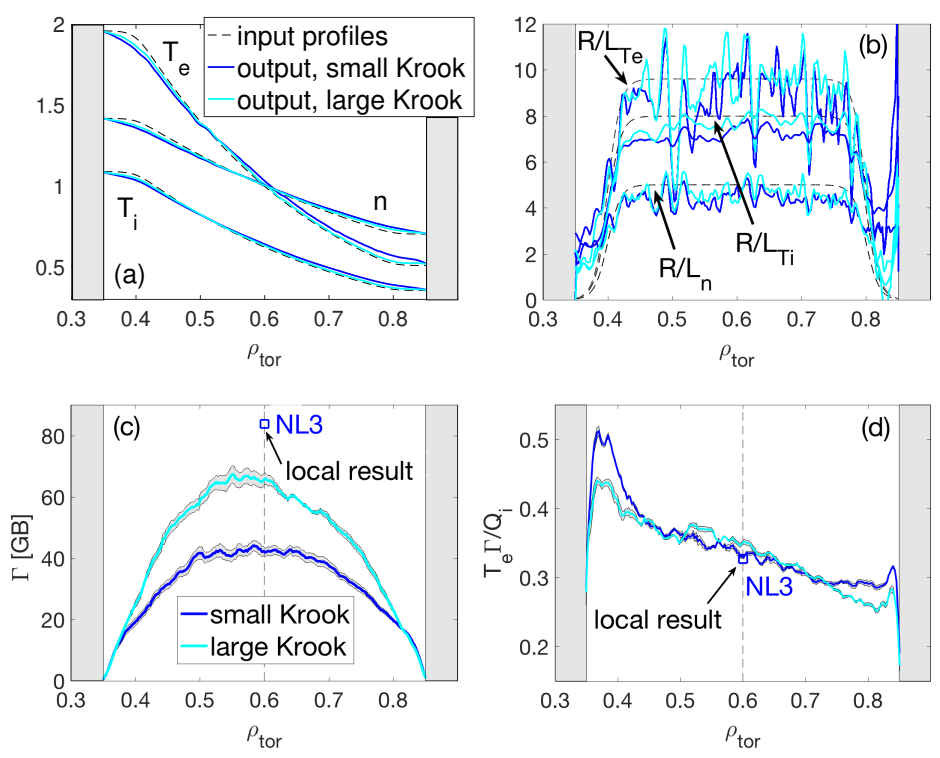

Figure 12. (color online) (a) Density profiles and (b) corresponding logarithmic gradients averaged over the last $\sim 30 R / c_{s}$ of the two global NL simulations which have been run varying the relaxation rates of the Krook-like sources. The temperature profiles are given in $T_{e}\left(\rho_{\text {tor }}=0.6\right)$ units, while the density profiles in $n\left(\rho_{\text {tor }}=0.6\right)$ units. The simulations inputs are indicated by black dashed lines. (c) Output particle fluxes in GB units, corresponding to input profiles from (a). The local value at $\rho_{\text {tor }}=0.6$ is indicated by a square marker for comparison. (d) Flux ratio $\Gamma[G B] / Q_{i}[G B]=T_{e} \Gamma / Q_{i}$, corresponding to input profiles from (a). The local value at $\rho_{\text {tor }}=0.6$ is indicated by a square marker for comparison.

Fig.12 (a) and (b) show the effect on density and temperature profiles when varying $\gamma_{H, P}$. The oscillations of the gradients are primarily due to profile corrugation. Analysing the corresponding particle flux outputs, an interesting behaviour has been found. Even if the absolute fluxes (represented in GB units in Fig.12 (c)) vary considerably when increasing $\gamma_{H, P}$, the flux ratios $\Gamma[G B] / Q_{i}[G B]=T_{e} \Gamma / Q_{i}$ are almost invariant close to the center of the radial box. Moreover, both global estimates of $T_{e} \Gamma / Q_{i}$ recover the local value at $\rho_{\text {tor }}=0.6$ (this last result is better illustrated in Appendix B, also con- sidering the global simulation with $R / L_{n}=3$ ). As a remark, in order to easily compare with Fig.9 (a), $\Gamma[G B]$ in Fig. 12 (c) is given in GB units at $\rho_{\text {tor }}=0.6$, following GENE normalisations, that is $\Gamma[G B]\left(\rho_{\text {tor }}\right)=\Gamma\left(\rho_{\text {tor }}\right) / \Gamma_{G B}\left(\rho_{\text {tor }}=\right.$ $0.6)$, while in Fig.12 (d), $\Gamma[G B]$ and $Q_{i}[G B]$ are given in $\mathrm{GB}$ units at each radius, that is $\left[\Gamma[G B] / Q_{i}[G B]\right]\left(\rho_{\text {tor }}\right)=$ $\left[\Gamma\left(\rho_{\text {tor }}\right) / \Gamma_{G B}\left(\rho_{\text {tor }}\right)\right] /\left[Q_{i}\left(\rho_{\text {tor }}\right) / Q_{i, G B}\left(\rho_{\text {tor }}\right)\right]$, which is equal to $T_{e}\left(\rho_{\text {tor }}\right) \Gamma\left(\rho_{\text {tor }}\right) / Q_{i}\left(\rho_{\text {tor }}\right)$.

\section{Appendix B: Global effects on flux ratios}

The analysis which has been pursued in section VB and presented in Fig.9 (a) has been repeated, comparing the flux ratio $\Gamma[G B] / Q_{i}[G B]=T_{e} \Gamma / Q_{i}$, instead of just $\Gamma[G B]$, with the local values at $\rho_{\text {tor }}=0.6$. The results are displayed in Fig.13.

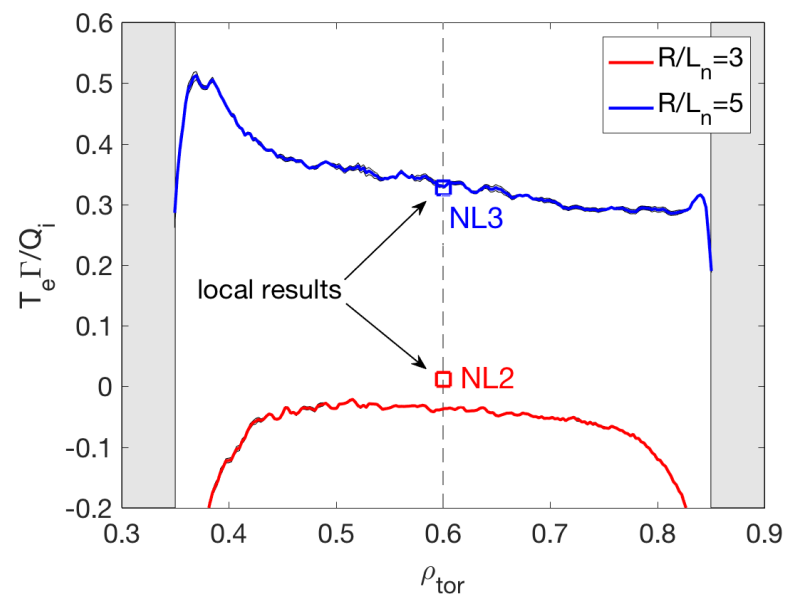

Figure 13. (color online) Flux ratio $\Gamma[G B] / Q_{i}[G B]=T_{e} \Gamma / Q_{i}$, corresponding to the two NL global simulations with $R / L_{n}=3,5$. The local values at $\rho_{\text {tor }}=0.6$ are indicated by square markers for comparison.

As can be seen, the large discrepancy between the global $\Gamma[G B]$ and the local value for the $R / L_{n}=5$ case disappears if we consider the particle flux to ion heat flux ratio instead. The small difference between the local and global values of $T_{e} \Gamma / Q_{i}$ for the $R / L_{n}=3$ case could be due to the different grid used in the local and global simulations or/and to the heavy electrons approximation which is considered in the latter case. Nevertheless, if we neglect this small difference, the global effects at $\rho_{\text {tor }}=0.6$ which were present if we looked at $\Gamma[G B]$, disappear if we consider the flux ratio $T_{e} \Gamma / Q_{i}$. Following this result, as a crosscheck, the global PF profile has been recomputed interpolating the density gradients with respect to $T_{e} \Gamma / Q_{i}$, instead of $\Gamma[G B]$ alone, at $T_{e} \Gamma / Q_{i}=0$. The resulting $\mathrm{PF}$ differs from the original one at most by $3 \%$ along the radius. 
[1] Special issue on ITER Physics Basis [Nucl. Fusion 39, 2175 (1999)].

[2] http://www.ipp.mpg.de/16355/demo

[3] C. Angioni, A. G. Peeters, F. Jenko, and T. Dannert, Phys. Plasmas 12, 112310 (2005).

[4] E. Fable, C. Angioni and O. Sauter, Plasma Phys. Control. Fusion 50, 115005 (2008).

[5] F. Merz and F. Jenko Nucl. Fusion 50, 054005 (2010).

[6] E. Fable, C. Angioni and O. Sauter, Plasma Phys. Control. Fusion 52, 015007 (2010).

[7] A. Skyman, D. Tegnered, H. Nordman and P. Strand Phys. Plasmas 21 , 092305 (2014).

[8] A. Mariani, S. Brunner, J. Dominski, A. Merle, G. Merlo, O. Sauter, T. Görler, F. Jenko, and D. Told Phys. Plasmas 25, 012313 (2018);

[9] F. Hofmann, J. B. Lister, M. Anton, S. Barry, R. Behn, S. Bernel, G. Besson, F. Bühlmann, R. Chavan, M. Corboz et al., Plasma Phys. Control. Fusion 36, B277 (1994).

[10] A. Bortolon, B. P. Duval, A. Pochelon, and A. Scarabosio, Phys. Rev. Lett. 97235003 (2006).

[11] B. P. Duval, A. Bortolon, A. Karpushov, R. A. Pitts, A. Pochelon, O. Sauter, A. Scarabosio, G. Turri and the TCV Team, Phys. Plasmas 15, 056113 (2008).

[12] F. Jenko, W. Dorland, M. Kotschenreuther and B. N. Rogers, Phys. Plasmas 7, 1904 (2000).

[13] O. Sauter, S. Brunner, D. Kim, G. Merlo, R. Behn, Y. Camenen, S. Coda, B. P. Duval, L. Federspiel, T. P. Goodman et al., Phys. Plasmas 21, 055906 (2014).

[14] Z. Lin, S. Ethier, T. S. Hahm, and W. M. Tang, Phys. Rev. Lett. 88, 195004 (2002).

[15] J. Candy, R. E. Waltz, and W. Dorland, Phys. Plasmas 11 (2004).
[16] B. F. McMillan, X. Lapillonne, S. Brunner, L. Villard, S. Jolliet, A. Bottino, T. Görler and F. Jenko, Phys. Rev. Lett. 105, 155001 (2010).

[17] G. Merlo, S. Brunner, O. Sauter, Y. Camenen, T. Görler, F. Jenko, A. Marinoni, D. Told and L. Villard, Plasma Phys. Control. Fusion 57, 054010 (2015).

[18] X. Lapillonne, S. Brunner, O. Sauter, L. Villard, E. Fable, T. Görler, F. Jenko and F. Merz, Plasma Phys. Control. Fusion 53, 054011 (2011).

[19] H. Luetjens, A. Bondeson, O. Sauter, Comput. Phys. Commun. 97, 219 (1999).

[20] T. Dannert and F. Jenko, Phys. Plasmas 12, 072309 (2005).

[21] F. Jenko, T. Dannert and C. Angioni, Plasma Phys. Control. Fusion 47, B195 (2005).

[22] F. Jenko and B. D. Scott, Phys. Plasmas 6, 2418 (1999).

[23] A. Casati, C. Bourdelle, X. Garbet, F. Imbeaux, J. Candy, F. Clairet, G. Dif-Pradalier, G. Falchetto, T. Gerbaud, V. Grandgirard et al. Nucl. Fusion 49, 085012 (2009).

[24] A. Mariani, G. Merlo, S. Brunner, A. Merle, O. Sauter, T. Görler, F. Jenko and D. Told J. Phys. Conf. Ser. 775, 012007 (2016).

[25] T. Görler, PhD Thesis: 'Multiscale Effects in Plasma Microturbulence', Universität Ulm (2009).

[26] T. Görler, X. Lapillonne, S. Brunner, T. Dannert, F. Jenko, F. Merz, et al., J. Comput. Phys. 230, 7053 (2011).

[27] D. Told, PhD Thesis: 'Gyrokinetic Microturbulence in Transport Barriers', Universität Ulm (2012).

[28] G. Merlo, PhD Thesis: 'Flux-tube and global grid-based gyrokinetic simulations of plasma microturbulence and comparisons with experimental TCV measurements', École Polytechnique Fédérale de Lausanne (EPFL) (2016). 